\title{
The parametric instability of resonance motion in restricted three body problem
}

\author{
Alexey E. Rosaev ${ }^{1}$ \\ ${ }^{1}$ NPC Nedra, Yaroslavl, Russia, \\ 150000 , Svobody str., $8 / 38$ \\ email: hegem@mail.ru
}

\begin{abstract}
A method of analyses of dynamical system is applied to the planetary restricted three-body problem (RTBP). It is well known, that equations of motion of restricted 3-body problem in rotating rectangular frame may be reduced to the second order differential equation with periodic coefficients (Hills equation). Here Hills equation in cylindrical coordinate frame is derived. It gives the possibility to estimate width and position of the unstable zones. The dependence of the position of unstable zones on orbital eccentricity of the test particle is derived. Some followings of this simple linear model are noted.
\end{abstract}

Keywords. celestial mechanics, methods: analytical, restricted three body problem, resonance, stability

\section{Introduction}

The three-body problem is a continuous source of study, since the discovery of its non-integrability due to Poincare (1892). However, some problems are still unresolved (Celletti et al.(2002)). In this paper, we consider the planar circular restricted threebody problem, when mass $m$ revolves around $M(M$ is much more than $m$ ) in a circular orbit and the third body is considered with negligible mass $\mathrm{m}_{0}$. We assume that all bodies move on the same plane. The equations of planar restricted Hill's problem in rectangular frame may be reduced to a Hill equation for normal distance from variation orbit x see Szebehely(1967). In our view, cylindrical system has some advantages over other ones, because the variation of one of the coordinates - central distance $\mathrm{R}$ - is always restricted and may be considered as a small parameter at the problem.

The main equations for the planar circular 3-body problem in cylindrical frame are:

$$
\begin{gathered}
\frac{d^{2}}{d t^{2}} \mathrm{R}-\mathrm{R}\left(\frac{d}{d t} \lambda\right)^{2}=\frac{\partial}{\partial R} U \\
\frac{d}{d t}\left(\mathrm{R}^{2} \frac{d}{d t} \lambda\right)=\frac{\partial}{\partial \lambda} U
\end{gathered}
$$

where the perturbation function $U$ corresponding to the heliocentric frame of reference is :

$$
U=\frac{G m}{D}+\frac{G M}{R}-\frac{G m R \cos (\delta \lambda)}{r^{2}}=\mathrm{U}(R, \lambda, t)
$$


where M - mass of the primary; $\mathrm{m}$ - mass of the perturbing body; $\mathrm{R}, \mathrm{r}$ - distance from the mass center test particle and perturbing body accordingly(the vector $r$ between the bodies of masses $\mathrm{M}$ and $\mathrm{m}$ being expressed as a function of $\mathrm{t}$ ); $\mathrm{G}$ - constant of gravity, $\delta \lambda$ - angle between perturbed and perturbing bodys(differential longitude of perturbed body), $\omega, \omega_{s}$ - mean motions perturbed and perturbing body accordingly, and:

$$
D=\sqrt{R^{2}+r^{2}-2 R r \cos (\delta \lambda)}
$$

There are two small parameters in problem: $\mathrm{x}$ - radial shift from intermediate (in variations) orbit and $\mathrm{r} / \mathrm{R}$ - the ratio of mean distance of perturbing and perturbed body. Let $\mathrm{R}(\mathrm{t})=R_{0}+\mathrm{x}(\mathrm{t})$, where $R_{0}$ is constant. In this paper, simple way to linearization of planar three body problem is considered. For study long-time evolution of orbits we consider averaged equations. In many averaged systems the second equation above is reduced to momentum $\mathrm{L}$ conservation. In this case we can rewrite first equation as:

$$
\frac{d^{2}}{d t^{2}} R-\frac{L^{2}}{R^{3}}=-\frac{G M}{R^{2}}+\frac{\partial}{\partial R} U
$$

\section{Simple linearization method}

Due to we have two small parameters, we can subsequently expand last expression by power $\mathrm{x}$ and $\mathrm{R} / \mathrm{r}$ ( or $\mathrm{R} / \mathrm{r}$ and $\mathrm{x}$ ) and restrict themselves by first order terms.

The principle part of expansion perturbation function may be written with using Legendres polynomials Stiefel\&Scheifele (1971). In case of the outer perturbing body it is possible to expand perturbation function by power $\mathrm{R} / \mathrm{r}$

$$
U=\frac{G M}{R}+\sum_{p=2}^{\infty} \frac{G m}{r}\left(\frac{R}{r}\right)^{p} P_{p}(\cos (\delta \lambda))
$$

After that, it is possible to transform this expansion into Fourier series. We obtain Hill's equation:

$$
\frac{d^{2}}{d t^{2}} x+\omega^{2} x=\mathrm{f}(t)
$$

where $\mathrm{f}(\mathrm{t})$ and $\omega$ depends on time.

Now we can easy take into account the eccentricity of perturbed particle orbit and show, that such generalization leads to very interesting results. At the elliptic osculating orbit, after the entering osculating orbital elements a - semimajor axis, e - eccentricity, for angular momentum L approximately:

$$
L=R^{2} \frac{d}{d t} \lambda=\sqrt{G M a\left(1-e^{2}\right)}
$$

$R_{0}$ depend on time. The phase-averaged value of $R_{0}$ approximately:

$$
R_{0}=\frac{1}{2} \pi \int_{0}^{2 \pi} \frac{a\left(1-e^{2}\right)}{1+e \cos (\phi)} d \phi=a \sqrt{1-e^{2}}
$$


After angular momentum substitution and linearization:

$$
3 \frac{L_{0}^{2}}{R_{0}{ }^{4}}-2 \frac{G M}{R_{0}{ }^{3}}=\frac{G M}{a^{3}}\left(1-\frac{3}{8} e^{4}\right)=\frac{G M}{a^{3}}(1-\alpha)
$$

Finally, for the probe particle elliptic motion case $\omega$ depends on eccentricity:

$$
\omega^{2}=\frac{G M}{a^{3}}\left(1-\frac{3}{8} e^{4}\right)+\sum_{p=2}^{\infty} \frac{G m}{r^{3}} p(p-1)\left(\frac{R}{r}\right)^{(p-2)} P_{p}(\cos (\delta \lambda))
$$

Then we can group terms with equal p $\delta \lambda$ by using Laplace's coefficients. The main frequency may be expressed:

$$
\omega^{2}=\frac{G M}{a^{3}}\left(1-\frac{3}{8} e^{4}\right)+\left(\sum_{p=2}^{\infty} \frac{G m b_{p} \cos (\delta \lambda)}{r^{3}}\right)
$$

where $b_{p}$ is easy to calculate numerically.

For investigation of stability, x may be negligible small. So, we can restrict only 1-st order in expansion by power $\mathrm{x}$. The Mathieu equation is a limit case of Hill's equation and it is more simply for studying. On the other side, because both equations (Hill and Mathieu) are linear, the main area of instability of Mathieu equation must be present in Hill's equation solution. Consequently, and in this case exist the areas of instability, complying with condition, see Landau\&Liphshitz (1973):

$$
\frac{\omega}{\omega_{s}}=\frac{n}{n-2+\alpha}
$$

Thereby received that orbits near resonances $(2 n+1) /(2 n-1)$ are unstable parametric. This conclusion completely coincides with results of the studies of the declared problem in the paper Hadjidemetriou(1982) by methods of matrix algebra. Directly, from view of linear equations in elliptic case, we can explain one interesting feature - centres of

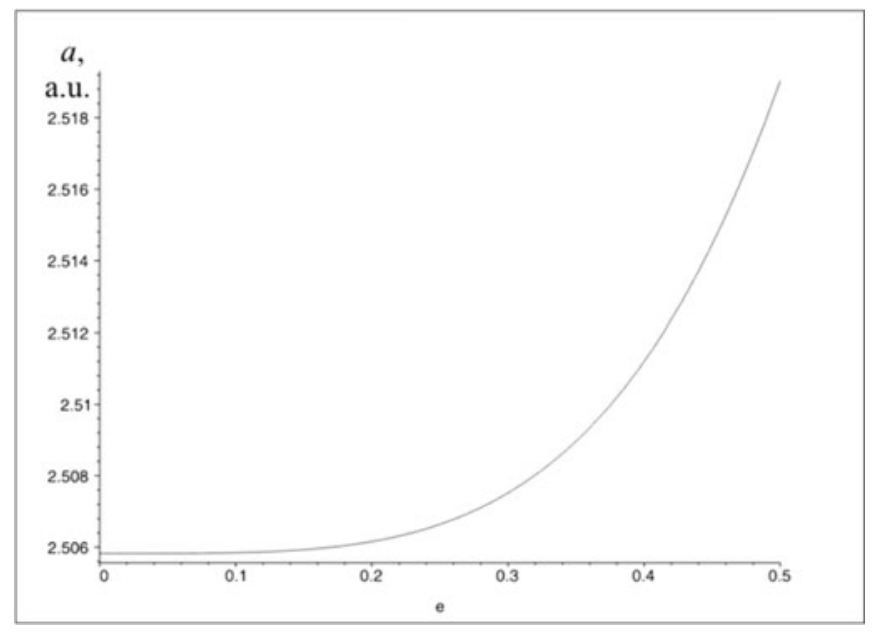

Figure 1. Position of exact resonance in dependence on the eccentricity for $3: 1$ commensurability with Jupiter. 
resonant zones are shifted out relative exact commensurability (resonance). In simple case one perturbing body, centres of instable resonant zones moved away from exact commensurabilities toward a source of perturbation (Fig. 1). So, exact commensurability may be out of the relatively according instable zone!

There are few another interesting applications of the presented simple linear average model. We note two of them. First, magnitude of eccentricity variations at resonance related with variations of semimajor axis. When eccentricity increase, semimajor axis decrease. As it is known (see Morbidelli (2002)), such behaviour is very common in resonance celestial mechanical systems. This relation can be explained in simple averaged linear model, but details are require special careful consideration and can be a subject of separate paper. Second, it is possible overlapping of unstable zones at high order of resonance.

\section{Conclusions}

It is known, that some different restricted three body problem modifications can be described by linear equation with time-dependent coefficients. Here we give the additional simplest method to obtain linear equation of motion. It is very suitable equation in variation to study perturbed motion at resonance. Just in a very simple model we can explain some very important characteristics of resonant motion. The main results of this work may be formulated in such form:

1. There are not exist stable orbits with $\mathrm{e}=0$ and $\mathrm{i}=0$ in neighbourhood of mean motion resonances $(2 \mathrm{n}-1) /(2 \mathrm{n}+1)$.

2. Positions and width of unstable zones depends on eccentricity. The results can be applied to a number of problems of Solar system dynamics. A number of minor planets on high elliptic resonance orbits show the regular behaviour of their orbits. We suppose that stability of these orbits can be provided due to large eccentricity.

In addition, some followings of this simple linear model are noted.

\section{References}

Celletti, A., Chessa, A., Hadjidemetriou, J., \& Valsecchi, G. B. 2002 Cel. Mech. Dyn. Astr. 83,239-255.

Morbidelli, A. 2002 Modern celestial mechanics. Taylor and Francis, 356

Hadjidemetriou, J. D.1982 Celestial Mechanics, 27, 305-322.

Landau, L. D. \& Liphshitz, E. M. 1973 Mechanics. Moscow, Nauka, 104-105.

Poincare, H. 1892 Les Méthodes Nouvelles de la Mécanique Celeste, Gauthier Villars, Paris.

Stiefel, E. L., \& Scheifele, G. 1971 Linear and Regular Celestial Mechanics. Springer-Verlag Berlin Heidelberg New York, 305.

Szebehely, V. 1967 Theory of Orbits. Acad.press New York and London. 